\title{
Development of a Method for Left Ventricular Shape Evaluation Based on Surfaces Obtained by Real-Time 3D Echocardiographic Images
}

\author{
F Maffessanti ${ }^{1}$, C Corsi $^{2}$, RM Lang ${ }^{3}$, EG Caiani $^{1}$ \\ ${ }^{1}$ Department of Biomedical Engineering, Polytechnic of Milan, Italy \\ ${ }^{2}$ DEIS, University of Bologna, Italy \\ ${ }^{3}$ Noninvasive Cardiac Imaging Lab, University of Chicago, IL, USA
}

\begin{abstract}
Left ventricular $(L V)$ shape is strictly related to $L V$ function. Previously proposed shape indexes suffer from their intrinsic $2 D$ nature. Our aim was to develop a method to quantify directly $3 D \mathrm{LV}$ shape from $\mathrm{LV}$ surfaces semi-automatically detected from real-time $3 D$ echocardiographic (RT3DE) images. First, a monodimensional shape descriptor has been obtained by sampling through an helical pattern the extracted endocardial surfaces. Then, four shape indexes have been defined by comparing the $L V$ with reference shapes.

The developed method was applied to RT3DE datasets obtained from 12 normal subjects, 8 patients with dilated cardiomiopathy and 7 ischemic patients. Some of the proposed shape indexes were able to follow chamber deformation throughout the cardiac cycle and to discriminate between normal and pathologic conditions.
\end{abstract}

\section{Introduction}

The mutual relationship between left ventricular (LV) function and shape is well known in literature. The relationship between LV dilation and an increase in LV globularity it's known, together with the reduction of ejection fraction (EF) connected with a decreased deformation of LV walls [1]. Alteration of the normal ellipsoidal LV cavity shape to a more spherical one was found to translate into an abnormal distribution of afterload, and associated with a higher early mortality rate in dilated cardiomyopathy patients [2].

The shape-function relationship is based on the physiological process of remodeling, that occurs in order to restore the normal LV function by inducing changes in LV shape. In the short term, this process results in functional restoration, but its stability is not lasting, resulting in a progressive worsening of the pathology.

In a common echocardiographic evaluation, however, while the LV function is quantified and used extensively as a prognostic index, no quantitative evaluation is routinely performed on LV shape. This is probably related to two intrinsic limitations: 1) the computation of global shape indexes supposes the knowledge about the position of the endocardium, which implies tedious and subjective manual tracing; 2) the potential inaccuracy in obtaining a global shape descriptor from a 2D projection, thus requiring geometric models. A first attempt in overcoming these limitations was based on the acquisition of multiple 2D views, usually by a transesophageal approach, and the application of shape indexes on the reconstructed 3D ventricle. However, this process implies multiple endocardial tracings and high computational effort.

We hypothesized that real-time three dimensional echocardiography (RT3DE), which allows the transthoracic acquisition of an entire cardiac volume, could represent the basis for overcoming these limitations, thus providing by semi-automated detection [3-4] the LV endocardial surfaces to be utilized for 3D shape analysis. Accordingly, the aim of this paper was to develop a method for the quantification of a new class of LV 3D shape indexes independent from geometrical modelling, and test their effectiveness in following LV deformation along the cardiac cycle, and in discriminating between normality and pathology.

\section{Methods}

Patients were enrolled at the University of Chicago Medical Center, Chicago, IL. A population of 27 subjects was studied, including 12 normal subjects $(\mathrm{N}$, mean age $34 \pm 8$ years), 8 patients with dilated cardiomyopathy (DCM, $57 \pm 17 \mathrm{yr}$ ), and 7 patients with regional wall motion abnormalities (RWMA, $65 \pm 11 \mathrm{yr}$ ).

RT3DE imaging was performed using a Sonos 7500 equipment (Philips Medical System, Andover, MA) with a 3000 active elements matrix array (X4 probe). Image 
acquisition was performed using wide-angle mode, in which 4 wedge shaped subvolumes (93 X 21 degrees) were obtained over 8 consecutive cardiac cycles during a breath hold with $\mathrm{R}$ wave gating. Moreover, in a subgroup of 12 patients (10 N, 2 DCM), cardiac magnetic resonance (CMR) images were obtained with a $1.5 \mathrm{~T}$ scanner (General Electric, Milwaukee, WI) with a phased array cardiac coil. Left ventricular endocardial surfaces were obtained from RT3DE datasets as described in [34]. CMR end-diastolic (ED) and -systolic (ES) volumes (V) were obtained by manual tracings (MASS analysis).

\subsection{Ventricular shape indexes}

First, a description of the 3D LV shape by a 1-D signal has been obtained by sampling through an helical pattern the LV surface [5].
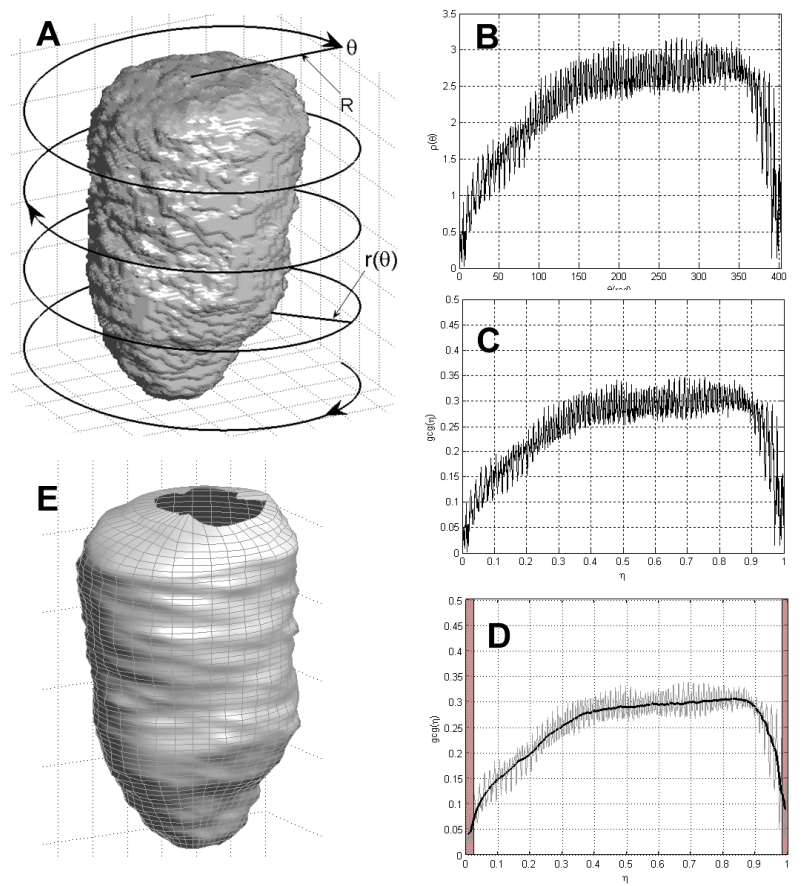

Figure 1. A) 3D dataset and the sampling helix; B) $\rho(\theta)$, representing the distance between the long axis and the LV surface; C) normalized descriptor $g c g(\eta)$; D) smoothed $g c g(\eta)$; E) reconstructed mesh

The relevant algorithm steps, represented in Figure 1, are the following: a) the 3D binary dataset is referred to its principal inertia axis, creating a floating reference system for each frame; b) an helical curve (64 windings, 36 samples per windings) wrapped around a cylindrical surface of radius $\mathrm{R}$, containing the $\mathrm{LV}$ and aligned along with the LV long axis (LAX), is introduced (Fig. 1.A); c) the function $\rho(\theta)$, describing the distance between LAX and the LV surface from base to apex along the helical curve, is calculated (Fig. 1.B) as :

$$
\rho(\theta)=R-r(\theta)
$$

where $\operatorname{r}(\theta)$ is the distance of the LV surface from the helical curve.

In order to allow the obtained shape descriptor to be dimensionless, independent by the size of LV, but able to discriminate between different aspect ratios (Figure 2), the following modifications were introduced to the original algorithm: I) the angular coordinate $\eta$, ranging from 0 to 1 , was obtained normalizing $\theta$ by its maximum value $\left(\theta_{\max }=64 \cdot 2 \pi\right)$; II) the shape descriptor, known as geometrical cardiogram, $g c g(\eta)$, was obtained normalizing $\rho(\eta)$ by LAX length (Fig. 1.C); III) the $g c g(\eta)$ was smoothed with a low-pass filter and its very end samples were excluded from the analysis to prevent potential apical or basal artifacts from introducing a bias the results (Fig 1.D).

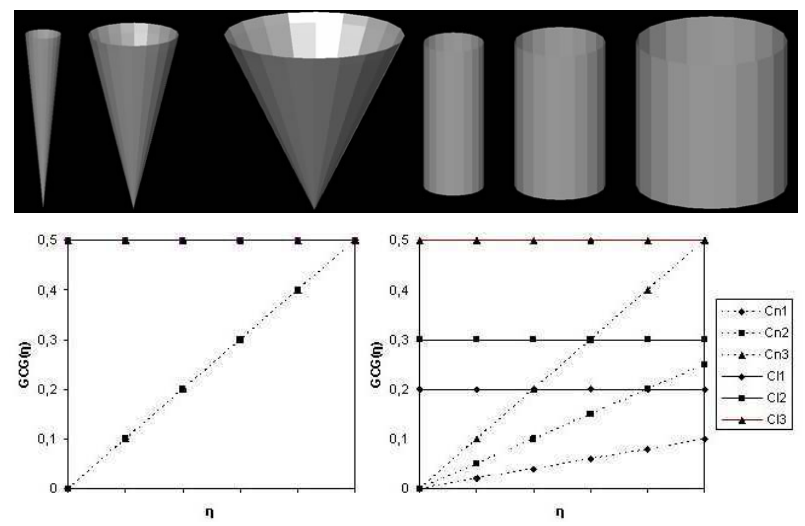

Figure 2. Comparison between the original ([5]) and the modified descriptor applied to a series of cones $(\mathrm{Cn} 1$, $\mathrm{Cn} 2, \mathrm{Cn} 3)$ and cylinders $(\mathrm{Cl} 1, \mathrm{Cl} 2, \mathrm{Cl} 3)$. The original method (left) discriminate between shapes but not between different aspect ratios, while the proposed method (right) allows this discrimination.

Once the $\operatorname{gcg}(\eta)$ signal is defined, a global shape index can be defined by measuring the degree of dissimilarity between the $g c g(\eta)$ computed from the LV and that obtained from a reference 3D shape:

$$
\text { Shape Index }=1-A / A_{\max }
$$

where $\mathrm{A}$ is the area between the two signals, and $\mathrm{A}_{\max }$ is the total area of the (gcg, $\eta$ ) plane (Figure 3).

The reference shapes we considered were: sphere ellipsoid, elliptical cone and cylinder. From these shapes, four global shape indexes were computed: spherical (S), ellipsoidal (E), conical (Cn) and cylindrical (Cl). 


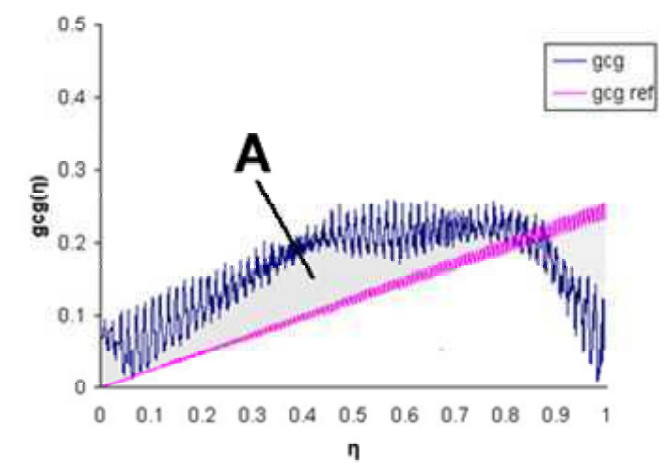

Figure 3. The area A (in grey) between the $g c g$ signal of the LV (in blue) and that of the reference shape (a cone in the example, in pink) measures the degree of dissimilarity.

\subsection{Statistical analysis}

First, linear regression analysis and Pearson correlation coefficient (r) between shape indexes and EDV, ESV volumes and EF obtained by CMR were computed. Then, separately for each patient population, a paired Student t-test $(\mathrm{p}<0.05)$ was applied to test for differences in the shape indexes between ED and ES.
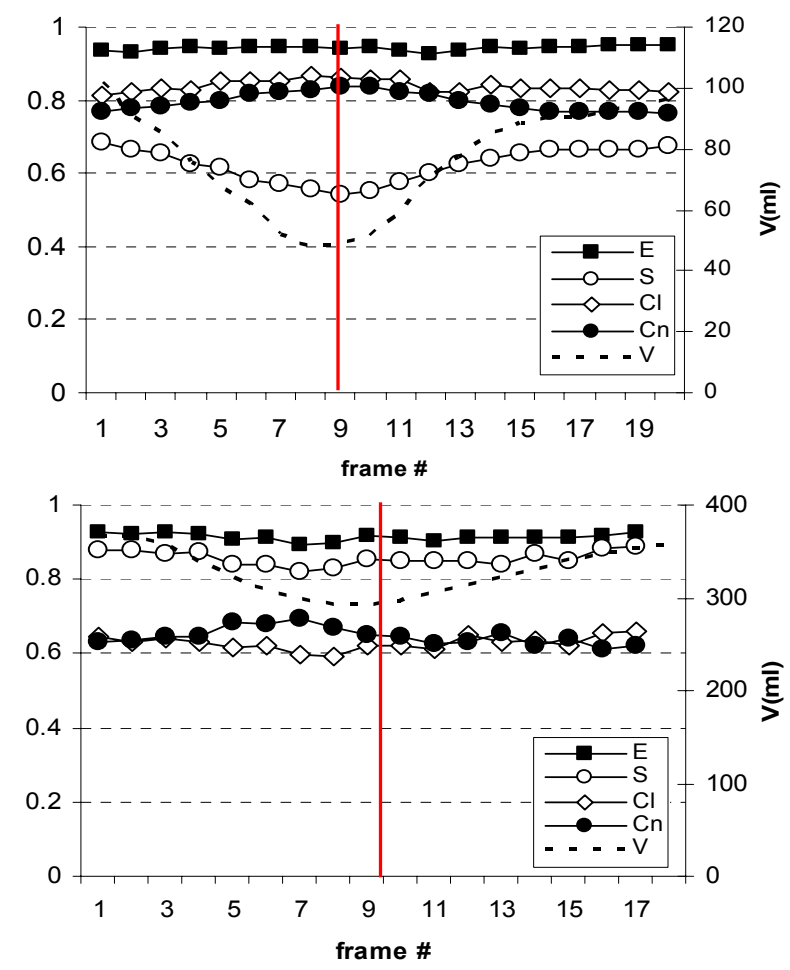

Figure 4. Values of the computed shape indexes throughout the cardiac cycle in a normal subject (top) and in a DCM patient (bottom). Vertical lines mark ES frames, while dotted lines represent LV volume (V).
Finally, one way ANOVA with Tukey test $(\mathrm{p}<0.05)$ was applied to evaluate shape differences between $\mathrm{N}$, DCM and RWMA. For those indexes showing noticeable differences, receiver operating characteristic (ROC) analysis was performed in order to define a threshold and test for its accuracy in discriminating between normality and pathology.

\section{Results}

\subsection{Correlation with CMR volumes}

The following significant correlations $(p<0.05)$ were found between the computed indexes and the LV dimensions and EF obtained from CMR: 1) S index had a positive correlation with both $\operatorname{EDV}(y=.0009 x+.5196$, $\mathrm{r}=.84)$ and $\operatorname{ESV}(\mathrm{y}=.0012 \mathrm{x}+.4991, \mathrm{r}=.91)$, while resulted negatively correlated with EF $(\mathrm{y}=-0.0041 \mathrm{x}+.8443, \mathrm{r}=-$ 0.808); 2) E index showed no significant correlation; 3) $\mathrm{Cn}$ index appeared negatively correlated to both EDV $(\mathrm{y}=-.0005 \mathrm{x}+.8612, \mathrm{r}=-.75)$ and $\operatorname{ESV}(\mathrm{y}=-.0008 \mathrm{x}+.8814$, $\mathrm{r}=-.95)$, and positively correlated to $\mathrm{EF}$ $(\mathrm{y}=.0025 \mathrm{x}+.6661, \mathrm{r}=.78) ; 4) \mathrm{Cl}$ index was negatively correlated to both $\operatorname{EDV}(y=-.0008 x+.9381, r=-.77)$ and ESV $(y=-.0009 x+.8732, r=-.63)$. No correlation with stroke volume was found in every index.

\subsection{Shape changes during cardiac cycle}

A representative example of the values in the shape indexes computed along one cardiac cycle in a normal subject and in a patient with DCM are shown in figure 4. Results evidenced that in the normal subjects the LV becomes less spherical during systole (ED: .64土.03, ES: $.58 \pm .06, \mathrm{p}<.01$ ), and more cylindrical (ED: .82+.06, ES: $.83 \pm .07, \mathrm{p}<.001)$. Conversely, changes in spherical shape index in the DCM patients were still present (ED: $.73 \pm .07, \mathrm{ES}: .70 \pm .07, \mathrm{p}<.05)$ but less evident. In RWMA

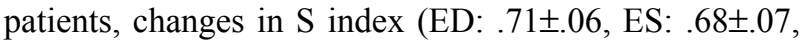

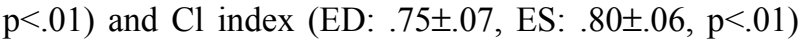
were noticed. Other indexes did not show significant differences between ED and ES frames.

\subsection{Intergroup differences}

The results of the comparison among the analyzed groups, separately for ED and ES frames, are shown in Figure 5 While the ellipsoidal index did not show any difference, the sphericity index S, as expected, was found significantly increased in DCM and RWMA patients both at ED and ES, compared to N. In addition, the conicity index $\mathrm{Cn}$ at ES was reduced in both DCM

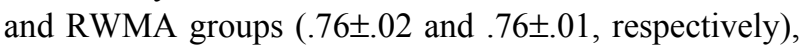
compared to $\mathrm{N}(.83 \pm .01)$. 

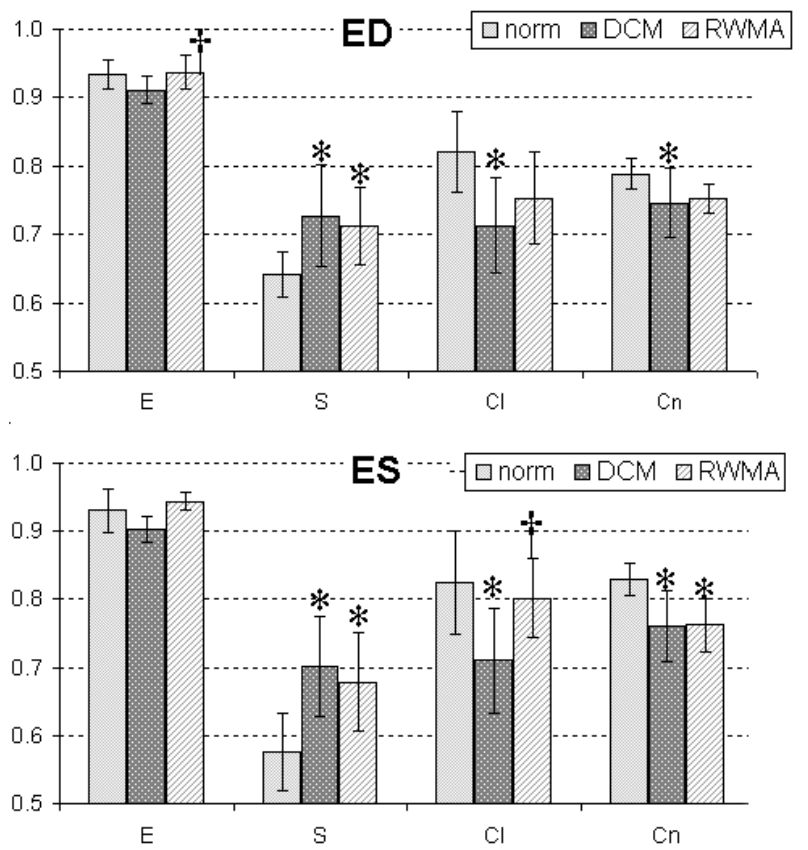

Figure 5. Shape indexes measured in $\mathrm{N}$ subjects (light gray), DCM (dark gray) and RWMA (with diagonal lines) patients, at end-diastole (left panel) and endsystole (right panel). Values expressed as mean $\pm \mathrm{SD} ; *$ : $\mathrm{p}<0.05$ vs N; $\uparrow: \mathrm{p}<0.05$ RWMA vs DCM.

\subsection{ROC analysis}

For the $\mathrm{S}$ and $\mathrm{Cn}$ shape indexes, ROC analysis was performed to determine their cut-off values, sensitivity $(\mathrm{Se})$, specificity (Sp), accuracy (Acc) in discriminating between normal and pathologic shape (Table 1). Values at end-systole showed the best performance; in particular the conicity index showed higher specificity and accuracy.

Table 1. Results of ROC analysis for sphericity and conicity index.

\begin{tabular}{cccccc}
\hline & & cut-off & Sp & Se & Acc \\
\hline \multirow{2}{*}{ S } & ED & 0.67 & 0.80 & 0.75 & 0.78 \\
& ES & 0.62 & 0.87 & 0.83 & 0.85 \\
\hline \multirow{2}{*}{ Cn } & ED & 0.76 & 0.60 & 0.92 & 0.74 \\
& ES & 0.80 & 0.93 & 0.83 & 0.89 \\
\hline
\end{tabular}

\section{Discussion and conclusions}

This paper represents the first attempt in computing global 3D LV shape indexes from RT3DE datasets. The proposed method allowed the automated quantification of several newly proposed shape indexes. The 3D LV shape information was exploited by reducing the problem complexity to $1-\mathrm{D}$, taking into account the LV shape ratio and without introducing any geometric assumption, thus overcoming the limitations related to previous shape indexes computations. The obtained results, in comparison with CMR data, confirmed the expected relationship between LV function and shape, with a reduction in EF related to an increase in sphericity index, and to a reduction in conicity index. Comparison between ED and ES indexes showed a reduced change in shape in pathologic subjects throughout the cardiac cycle, compared to normally shaped ventricles. Sphericity and conicity indexes at end-systole resulted in high accuracy in discriminating between $\mathrm{N}$ and pathological ventricles, showing the potential utility of these indexes in clinical practice. In particular, they could be utilized in conjunction with EF to assess the effects of pharmacological or surgical treatments in follow-up protocols. Their potential in early detection of LV shape abnormalities that could precede LV function worsening needs to be tested in specific protocols. Moreover, this method can be applied to LV surfaces obtained from other medical imaging modalities.

\section{References}

[1] Lewis RP, Sandler H. Relationship between changes in left ventricular dimensions and the ejection fraction in man. Circulation 1971;44:548-57

[2] Douglas PM, Morrow R, Ioli A, Reichek N. Left ventricular shape, afterload and survival in idiopathic dilated cardiomyopathy. J Am Coll Cardiol 1989;13:311-5.

[3] Caiani EG, Corsi C, Zamorano J, et al. Improved semiautomated quantification of left ventricular volumes and ejection fraction using 3-D echocardiography with a full matrix array transducer: comparison with magnetic resonance imaging. J Am Soc Echocardiogr 2005;8:779-88

[4] Corsi C Lang RM, Veronesi F, et al. Volumetric quantification of global and regional left ventricular function from real-time three dimensional echocardiographic images. Circulation 2005;112:1161-70

[5] Azhari H, Sideman S, Beyar R, et al. An analytical descriptor of three dimensional geometry: application to the analysis of the left ventricular shape and contraction. IEEE Trans Biomed Eng, 1987;34:345-55

Address for correspondence

Dr Enrico Caiani

Dipartimento di Bioingegneria

Politecnico di Milano

Piazza L. da Vinci 32, Milano, Italy

E-mail: caiani@biomed.polimi.it 